\title{
Sonografische Lymphknotendiagnostik im Bereich der unteren Extremitäten
}

\section{Ultrasound lymph node examination of the lower extremities}

Autor

Hans-Peter Weskott

Institut

Ultraschall Ambulanz am Klinikum Siloah Hannover

Schlüsselwörter

Lymphknoten, Differenzialdiagnose, untere Extremität

Key words

lymph nodes, differential diagnosis, lower extremity

Bibliografie

Phlebologie 2020; 49: 351-362

DOI 10.1055/a-1247-5486

ISSN 0939-978X

(c) 2020. Thieme. All rights reserved.

Georg Thieme Verlag KG, Rüdigerstraße 14,

70469 Stuttgart, Germany

Korrespondenzadresse

Dr. Hans-Peter Weskott

Ultraschall Ambulanz am Klinikum Siloah Hannover,

Roesebeckstr. 15, 30449 Hannover, Deutschland

weskotthp@t-online.de

\section{ZUSAMMENFASSUNG}

Die Sonografie dient der Detektion und Charakterisierung tastbarer Resistenzen oder zufällig entdeckter Lymphknoten (LK) der unteren Extremitäten. Meist handelt es sich um chronisch entzündliche oder reaktive Lymphknoten ohne klinische Relevanz. Sie finden sich fast immer nur inguinal, während LK in der Kniekehle sehr selten auftreten. Für die Differenzialdiagnose kommt neben Anamnese und Klinik der B-Bild-Sonografie sowie der Gefäßarchitektur eine wegweisende Rolle zu. Wegen der unspezifischen sonografischen Befunde kann

bei einem singulären LK nicht sicher zwischen entzündlichen und Lymphomerkrankungen unterschieden werden: So findet sich eine Rindenverdickung bei erhaltener Gefäßarchitektur sowohl bei Lymphomen als auch bei entzündlich-reaktiven LK. Eine US-gezielte Biopsie kann diagnostisch wegweisend sein. Eine metastatische Transformation geht sonografisch oft mit einer Zerstörung der LK-Architektur und des geordneten Gefäßbildes einher. Wichtig sind ferner die LK-Abgrenzbarkeit und die zentrale Ischämie in der farbkodierten und kontrastverstärkten Sonografie.

\section{ABSTRACT}

Sonography is used to detect and characterize palpable resistance or accidentally discovered lymph nodes (LN) of the lower extremities. In most cases, these are chronically inflammatory or reactive lymph nodes without clinical relevance. They are almost always found only in the groin, while LN only occur very rarely in the popliteal. In addition to the patient's medical history and clinic, B-scan sonography and vascular architecture also play a decisive role in differential diagnosis. Due to the unspecific sonographic findings, it is not always possible to differentiate reliably between inflammatory and lymphoma diseases in a singular LN: Therefore, a thickening of the cortex with preserved vascular architecture, for example, is found in lymphomas as well as in inflammation-reactive LN. An US targeted biopsy can be diagnostically helpful. A metastatic transformation often goes hand in hand with the destruction of the $L N$ architecture and the orderly vascular image. Also important is the $\mathrm{LN}$ delimitation and central ischemia in the color-coded and contrast-enhanced sonography.

\section{Einführung, funktionelle Anatomie}

Es wird geschätzt, dass in 24 h etwa 2 Liter Lymphflüssigkeit im Gewebe gebildet werden, die über offene Enden von Lymphgefäßen zusammen mit immunkompetenten Zellen weiter zu den nächstgelegenen LK transportiert werden. Die Lymphgefäße haben ein feines Klappensystem und verlaufen parallel zu den Gefäßen. Die Lymphe tritt über mehrere afferente Lymphbahnen in die von Septen begrenzten funktionellen Kompartimente eines LK ein. Bei der Lymphknotenpassage wird die Lymphe von als fremd erkannten Antigenen gefiltert ( $\triangleright$ Abb. 1a). Die so „gereinigte“ Lymphe gelangt über eine bis wenige efferente Lymphbahnen weiter nach proximal ( $\triangleright$ Abb. 1 b). Nach Passage weiterer LK gelangt sie in die abdominellen und thorakalen Lymphbahnen, von wo aus sie schließlich wieder dem zentralen Venensystem zugeführt wird [1].

Von den geschätzten 300-700 Lymphkoten findet sich etwa ein Drittel im Halsbereich (häufige Eintrittspforte für Krankheitserreger). Daher können mit Ultraschall bei jedem Menschen LK im Halsbereich detektiert werden. Auch inguinal finden sich 


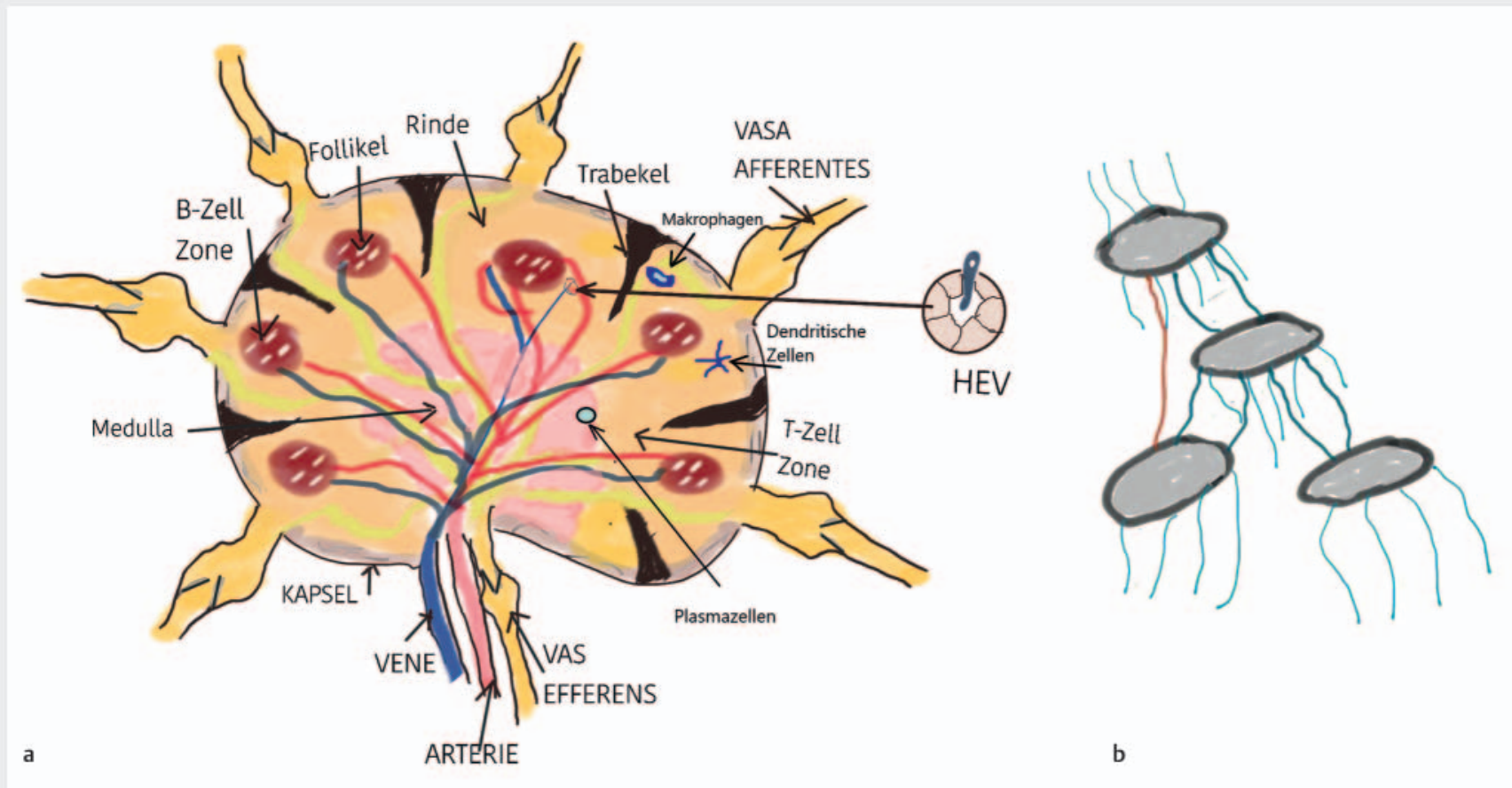

- Abb. 1 a Vereinfachtes Schema eines Lymphknotenaufbaus. Die einströmende Lymphe wird auf mehrere Kompartimente (Raum zwischen zwei Trabekeln) verteilt; nach Filterung erfolgt die Drainage über einen bis wenige efferente Lymphbahnen. In der äußeren Rindenschicht finden sich die Follikel (B-Zellen), in der inneren die T-Lymphozyten. Die versorgenden Gefäße finden sich im Hilus. In der Endstrecke von Arterien und Venen finden sich die HEV (High Endothelial Venules), über die immunkompetente Zellen aus den Gefäßen in das LK-Gewebe austreten. Dort werden Antigene von dendritischen Zellen festgehalten und den aktivierten Lymphozyten zur zellulären und humoralen Elimination präsentiert. $\mathbf{b}$. Lymphografiebild der afferenten und efferenten Lymphbahnen, die rot markierte Linie entspricht einen LK umgehenden Lymphgefäß [2].

bei fast allen Menschen LK, vorausgesetzt, geeignete Schallköpfe kommen zum Einsatz.

Ein intakter Lymphknoten besteht aus Rinde, Mark und Hilusregion und wird von einer Kapsel ummantelt. Gelegentlich kann man mit hochfrequenten Schallköpfen kleine echoarme Follikel in entzündlichen oder Lymphom-LK darstellen ( $\mathbf{A b b}$. 3). Im Hilus finden sich versorgende Gefäße sowie das abführende Lymphgefäß.

\section{Ultraschallwerkzeuge, Untersuchungsablauf}

In der Peripherie werden i.d. R. lineare Schallköpfe zwischen 7,5 und $20 \mathrm{MHz}$ eingesetzt. Je flacher die Lage der LK, desto höher sollte die Schallkopffrequenz gewählt werden. Neben dem B-Mode kommt die farbkodierte Duplexsonografie (FKDS) regelhaft zum Einsatz, ggf. auch von einigen Firmen patentierte Flussdetektionsverfahren wie B-Flow (Darstellung von fließendem Blut als leuchtend weiße Punkte durch Diskriminierung stationärer Echos von sich bewegenden Echos) und SMI (Superb Microvascular imaging mit artefaktreduzierter FKDS) [3-5]. Nur in Ausnahmefällen wird die Elastografie oder die kontrastverstärkte Sonografie (CEUS = Contrast-Enhanced Ultrasound = Ultraschall mit Kontrastmittelverstärkung, $\mathbf{A b b}$.4b) eingesetzt [6]. Aus einem eingefrorenen Gewebeblock lässt sich ggf. eine C-Ebene oder ein Panoramabild ( $\bullet$ Abb. 2) generieren; die Schnittebene liegt dann parallel zur Kutis ( $\triangleright \mathbf{A b b} \mathbf{2}, \mathbf{6 b}$ ). Für eine oft auch endgültige Diagnosestellung wird die Sonografie für eine gezielte Feinnadela-

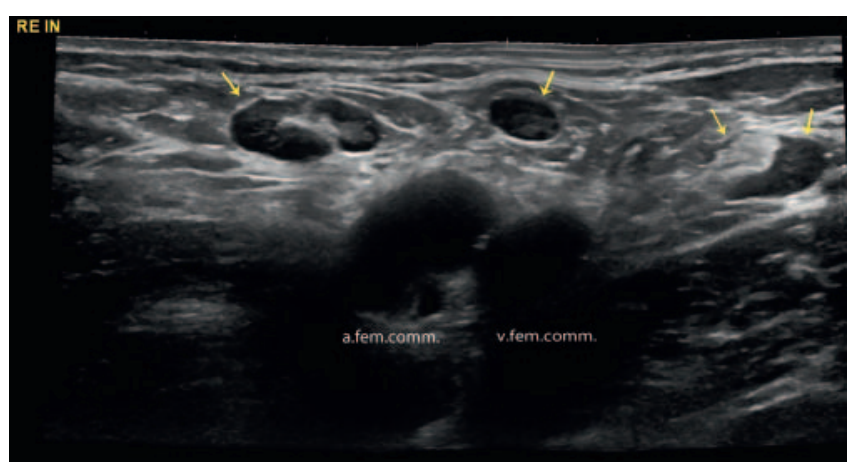

- Abb. 2 Horizontal liegende, durch Non-Hodgkin Lymphome (NHL) transformierte Lymphknoten (gelbe Pfeile). Der linke LK weist eine deutlich verdickte Rinde auf, der mittlere lässt kein echogenes Zentrum erkennen, der rechte LK hat eine noch erhaltene dünne Rinde, während der mediale Anteil deutlich echoarm verdickt ist. Dorsal liegen die im Schrägschnitt getroffenen Femoralgefäße.

spirationspunktion (FNAP) oder Schneidbiopsie eingesetzt. Vor allem bei der definitiven Typisierung von Lymphomen wird jedoch eine LK-Extirpation empfohlen. Eine elektive Lymphadenektomie des Wächter-LK wird gemäß AWMF-Leitlinien nicht empfohlen $[7,8]$ ( Tab. 1).

Die Untersuchung erfolgt zunächst entlang des Leistenbandes und der proximalen großen Gefäße im Querschnitt mit einem den anatomischen Gegebenheiten angepassten, möglichst hochfrequenten linearen Schallkopf. Wird ein LK gefunden, muss dieser 

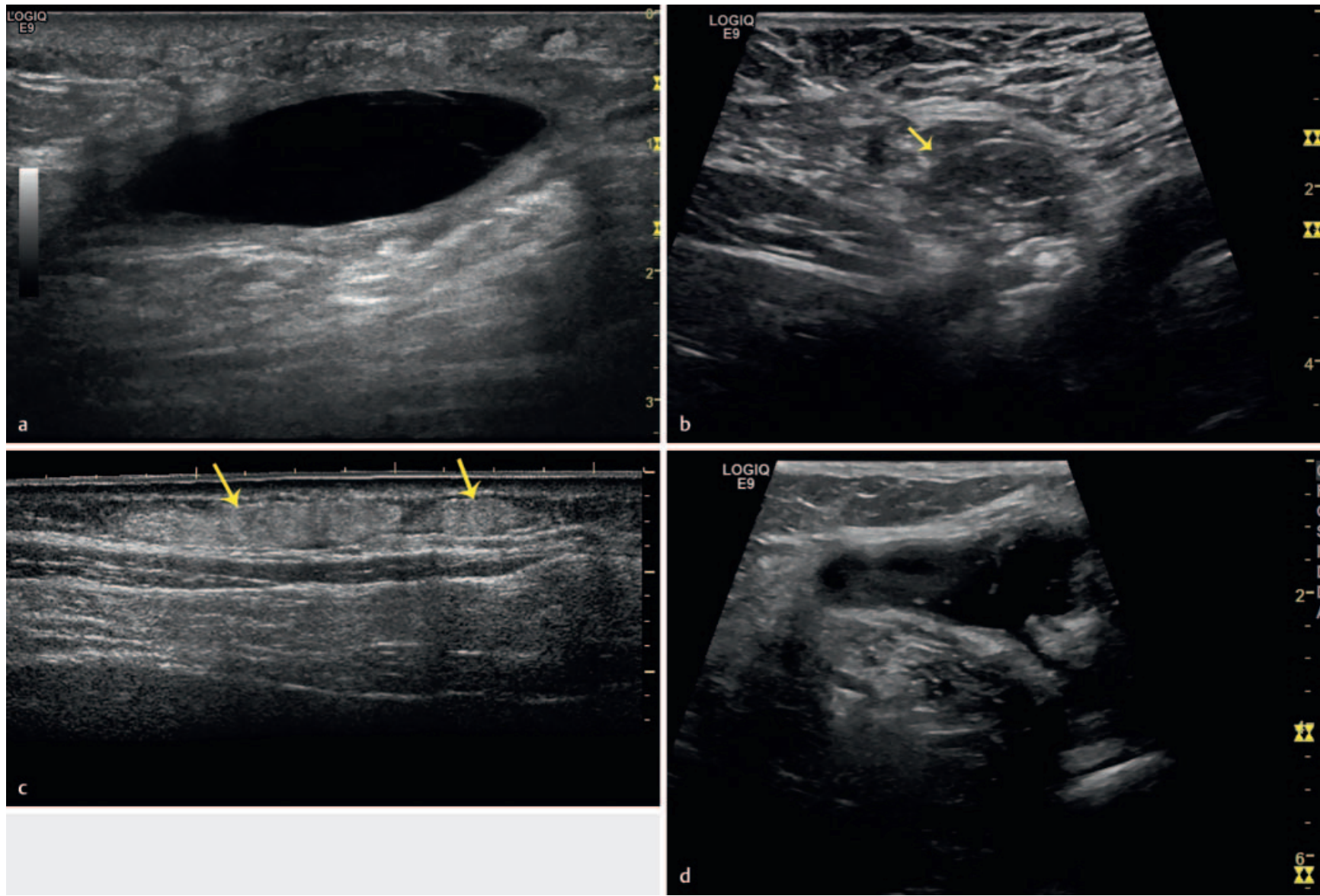

- Abb. 3 a Postoperatives Serom mit Umgebungsödem in der Leistenregion. b Bakerzyste (Kniegelenk). c Inguinale Hernie (Pfeil) nach ValsalvaManöver. d Subkutane tastbare Lipome in der FKDS ohne Vaskularisation (Pfeile).

in zwei Ebenen vermessen werden; dem Quotienten zwischen Längs- und Querdurchmesser (Solbiati- bzw. Vassallo-Index) kann bei metastatischen LK eine allerdings eingeschränkte differenzialdiagnostische Bedeutung zukommen ( $\bullet$ Tab. 2): Liegt der Quotient unter 2, ist eine metastatische Genese nachzuweisen bzw. auszuschließen [9, 10]. Längliche LK mit einem Solbiati-Index $>2$ sind jedoch hinsichtlich der Dignität wenig spezifisch. Es folgt ein Sweep in beiden Schallachsen. Im B-Mode ist auf die scharfe Abgrenzbarkeit zur Umgebung hin zu achten. Die FKDS kann mit einem empfindlichen Flussdetektionsverfahren die LK-Vaskularisation, die CEUS kann die Gewebeperfusion nachweisen [6, 11]. Diese Verfahren dienen auch der Darstellung der Gefäßarchitektur. Aufgaben der Bildgebung sind damit die Detektion und Lokalisation von LK, ihre Charakterisierung, ggf. Unterstützung bei der Punktion. Die CEUS kann Zusatzinformationen liefern, ist aber dem erfahrenen Untersucher und nur speziellen Fragestellungen vorbehalten [5]. Der differenzialdiagnostische Wert der Elastografieverfahren ist noch nicht abschließend geklärt.

\section{Lokalisation, häufige Differenzialdiagnosen}

Man unterscheidet oberflächliche horizontal, unmittelbar unter dem Leistenband nach lateral und medial gelegene, superolate- rale und superomediale LK ( $\bullet$ Abb. 2 ). Sie sind im Unterschied zu den tiefer liegenden LK oft tastbar. Etwas kaudal vom Hiatus saphenus liegen die oberflächlichen inferioren LK. Die tiefer gelegenen Lymphknoten finden sich subfaszial medial der Femoralgefäße (s. Brenner in diesem Heft). Ein wichtiger klinischer Parameter sind Größe und Schmerzempfindlichkeit von LK. Akut entzündliche LK sind im Gegensatz zu chronisch oder reaktiv entzündlichen LK meist tastbar oder druckschmerzhaft und führen den Patienten zum Arzt.

Lymphknoten in der Kniekehle sind sehr selten und in der Regel entzündlicher oder tumoröser Genese [12]. Sie finden sich meist bei distal gelegenen Entzündungen (z. B. Erysipel, Ulcus cruris) oder kutanen Malignomen [13].

Über die inguinalen LK wird die Lymphe aus den Beinen, dem äußeren Genitale und der Gesäßregion sowie zum anderen von der Kutis und Subkutis unterhalb des Nabels drainiert.

Häufige lokale Differenzialdiagnosen sind Lipome, Hernien, Gelenkergüsse, postoperative Serome und Hämatome ( Abb. 3a-d, 4a, b).

Echogene, meist subkutan gelegene Herde entsprechen meist palpablen Lipomen ( $\bullet \mathbf{A b b} \mathbf{3} \mathbf{d} \mathbf{d}, \mathbf{4 a}, \mathbf{b})$. Sie weisen in der FKDS keine Vaskularisation auf. Bei Wachstum oder aber Nachweis intraläsionaler Gefäße muss eine histologische Klärung erfolgen [14]. 

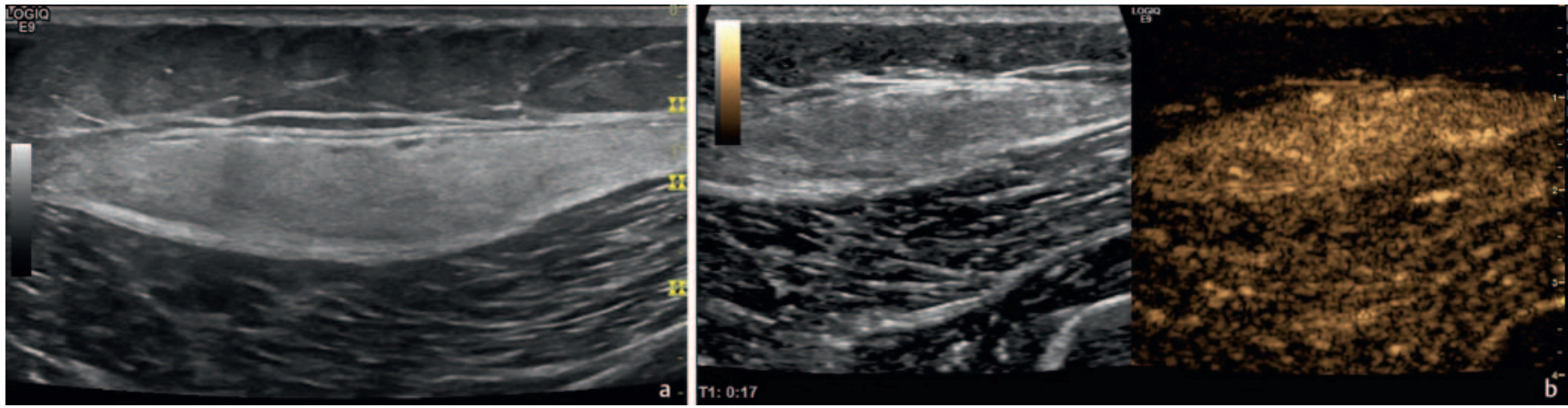

- Abb. 4 a Die tastbare Resistenz an der Innenseite des Oberschenkels auf dem M. adductor longus entspricht einem scharf begrenzten, echogenen, spindelartigen Lipom von $53 \mathrm{~mm} \times 9 \mathrm{~mm}$. b Wegen der deutlichen Vaskularisation in der FKDS und Hyperperfusion in der CEUS* erfolgte die operative Klärung (Histologie: braunes Fett, Hibernom) * Contrast-Enhanced Ultrasound - Ultraschall mit Kontrastmittelverstärkung.

- Tab. 1 Technische Ultraschallverfahren.

Technik, Beurteilungskriterien

\begin{tabular}{|c|c|c|c|}
\hline \multirow{2}{*}{$\begin{array}{l}\text { Schallkopfwahl } \\
\text { B-Mode: Fundamental, THI } \\
\text { (Tissue harmonic imaging) }\end{array}$} & \multicolumn{3}{|c|}{$\begin{array}{l}\text { Linearer Schallkopf, je oberflächlicher der LK, desto höher die Frequenz; die Frequenz für die Farbkodierung ist nied- } \\
\text { riger als im unterlegten B-Bild } \\
\text { Darstellungsmodi: C-Ebene, Extended Field of Use }\end{array}$} \\
\hline & $\begin{array}{l}\text { Anzahl, Seitendifferenz, lokaler Druck- } \\
\text { schmerz, Entfernung zum Primum }\end{array}$ & LK-Architektur: Rinde/Mark/Hilus & Kapsel intakt, lokales Ödem? \\
\hline $\begin{array}{l}\text { FKDS: Farbdoppler, Power- } \\
\text { Doppler, B-Flow, SMI }\end{array}$ & Intranodale Gefäße? Gefäßarchitektur & Arterien \& Venennachweis & Perinodale Gefäße \\
\hline $\begin{array}{l}\text { Elastografie (Strain- und } \\
\text { Scherwellen-E.) }\end{array}$ & $\begin{array}{l}\text { Farbkodierte Darstellung der lokalen } \\
\text { Gewebehärte }\end{array}$ & $\begin{array}{l}\text { Strain-E.; Cave: Nur Vergleichs- } \\
\text { werte werden angezeigt. }\end{array}$ & $\begin{array}{l}\text { Scherwellen-E.: Absolute lokale } \\
\text { Werte werden in } \mathrm{m} / \mathrm{s} \text { oder } \mathrm{kPa} \\
\text { angezeigt. }\end{array}$ \\
\hline CEUS & $\begin{array}{l}\text { Kontrastierungsrichtung: zentripetal, } \\
\text { zentrifugal }\end{array}$ & $\begin{array}{l}\text { Homogene/fehlende } \\
\text { Kontrastierung? }\end{array}$ & Perinodale Kontrastierung? \\
\hline
\end{tabular}

\section{Reaktiv-entzündliche Lymphknoten}

Inguinale Lymphknoten finden sich zumeist mit chronisch reaktiver bzw. entzündlicher Genese bei fast allen Menschen. Die Rinde kann sehr schmal sein $(<1 \mathrm{~mm})$, eine Vaskularisation ist mittels FKDS dann meist nicht darstellbar. Das Zentrum des LK ist i. d. R. echogen (Fettgewebe, s. - Abb. 5b, 6a-d), kann gelegentlich aber auch echoärmer als das den LK umgebende Fettgewebe sein ( $>$ Abb. 5b).

Mit zunehmender Entzündungsaktivität nehmen die Rindendicke (Follikel, Paracortex) und der Vaskularisationsgrad zu ( $\triangleright$ Abb.5a-d, 6c-e, 7a-c, 8). Eine regional begrenzte Auftreibung der Rinde sieht man, wenn die afferente Lymphbahn Antigene in die zuständigen Kompartimente des LK drainiert ( $>$ Abb. 1, 6a-e) und so eine regionale Immunantwort auslöst. Akut entzündliche LK finden sich meist unilateral.

Die benigne Lymphadenopathie ist oft selbstlimitierend.

Ätiologisch kommen neben akuten und chronisch entzündlichen Infektionen der Haut wie Psoriasis und Neurodermitis auch autoimmune Erkrankungen (z. B. rheumatoide Arthritis, Dermatomyositis, Lupus erythematosus), medikamentöse Therapien (z. B.
Interferontherapie) sowie iatrogene Ursachen (z. B. lokale Interventionen) infrage ( $\triangleright$ Abb.8a-f) $[14,15]$.

Die wichtigsten sonografischen Unterscheidungskriterien zwischen entzündlich reaktiven, metastatischen und nodalen Lymphomerkrankungen finden sich in $>$ Tab. 2. Dabei stellt die Größe von Lymphknoten allein kein Differenzierungsmerkmal dar.

Abszedierende entzündliche LK sind selten und kommen meist nur zervikal (z. B. Tuberkulose), inguinal bei venerischen Erkrankungen oder bei Immuninkompetenz vor.

\section{Metastatisch transformierte Lymphknoten}

Die Sonografie ist der Palpation bei der Detektion von Lymphknotenmetastasen deutlich überlegen [16]. Maligne Infiltrationen von Lymphknoten der unteren Extremität finden sich am häufigsten bei malignem Melanom, malignen Lymphomen, Plattenepithelkarzinomen des Analkanals, von Vulva und Penis, Sarkomen und kutanen Plattenepithelkarzinomen wie auch des Rumpfes. Da bei lymphatischer Metastasierung die Tumoren konzentrisch vom Randbereich oder Zentrum aus wachsen, erscheinen sie meist als asymmetrische, echoarme Rindenknoten oder als kugelige echoarme Lymphknotenherde. Initial bei hämatogener Metastasierung 

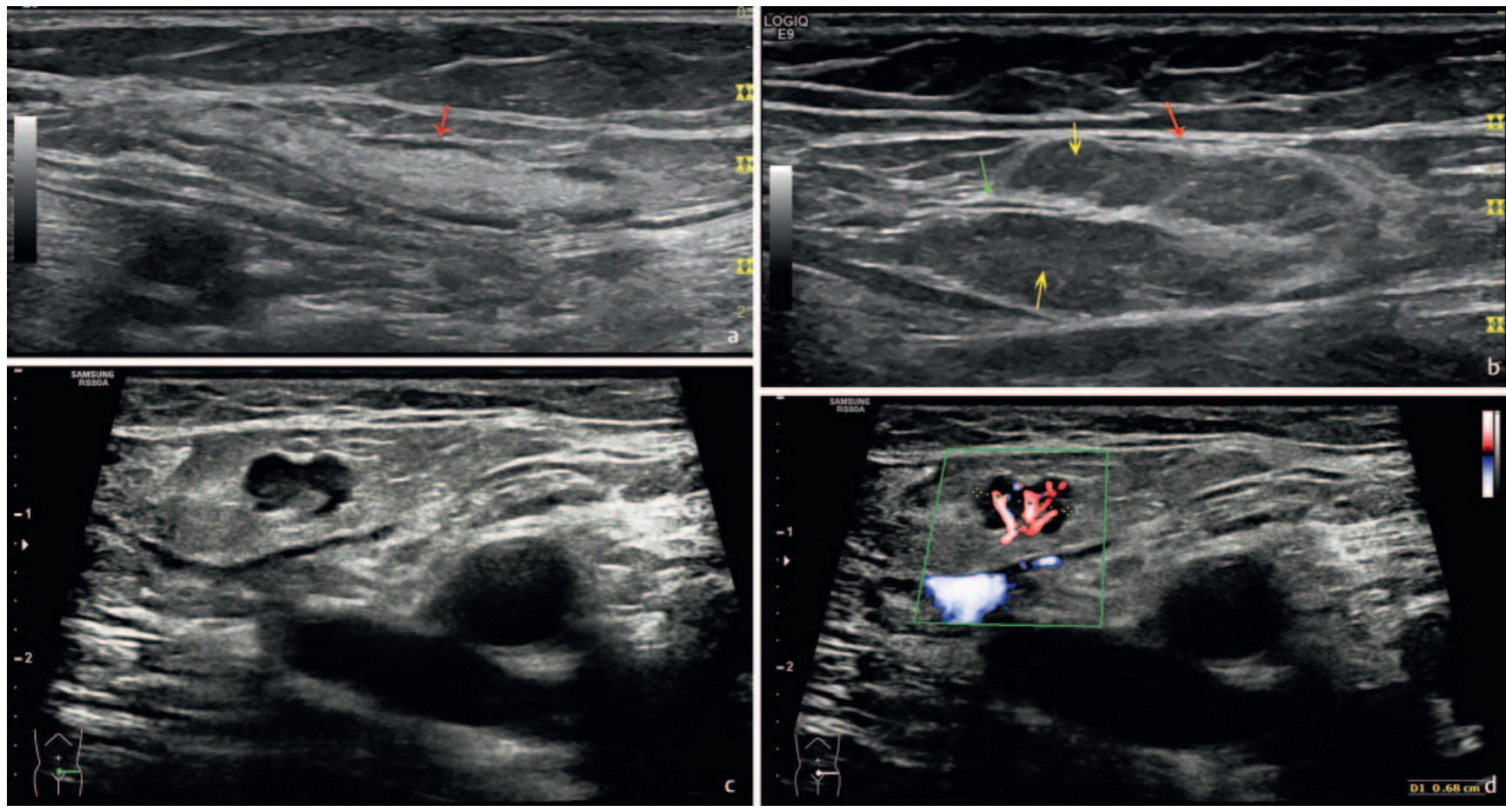

Abb. 5 a Zentral echogener (verfetteter), länglicher LK mit ca. 0,7 mm dicker Rinde (roter Pfeil). b Zentral echoärmere Medulla (gelbe Pfeile: Fettinfiltrate), roter Pfeil: ca. 0,6 mm schmaler Kortex. c, d 7 mm großer LK mit echoarmer, relativ zum echogenen Hilus verdickter Rinde b. In der FKDS geordnetes Gefäßbild.
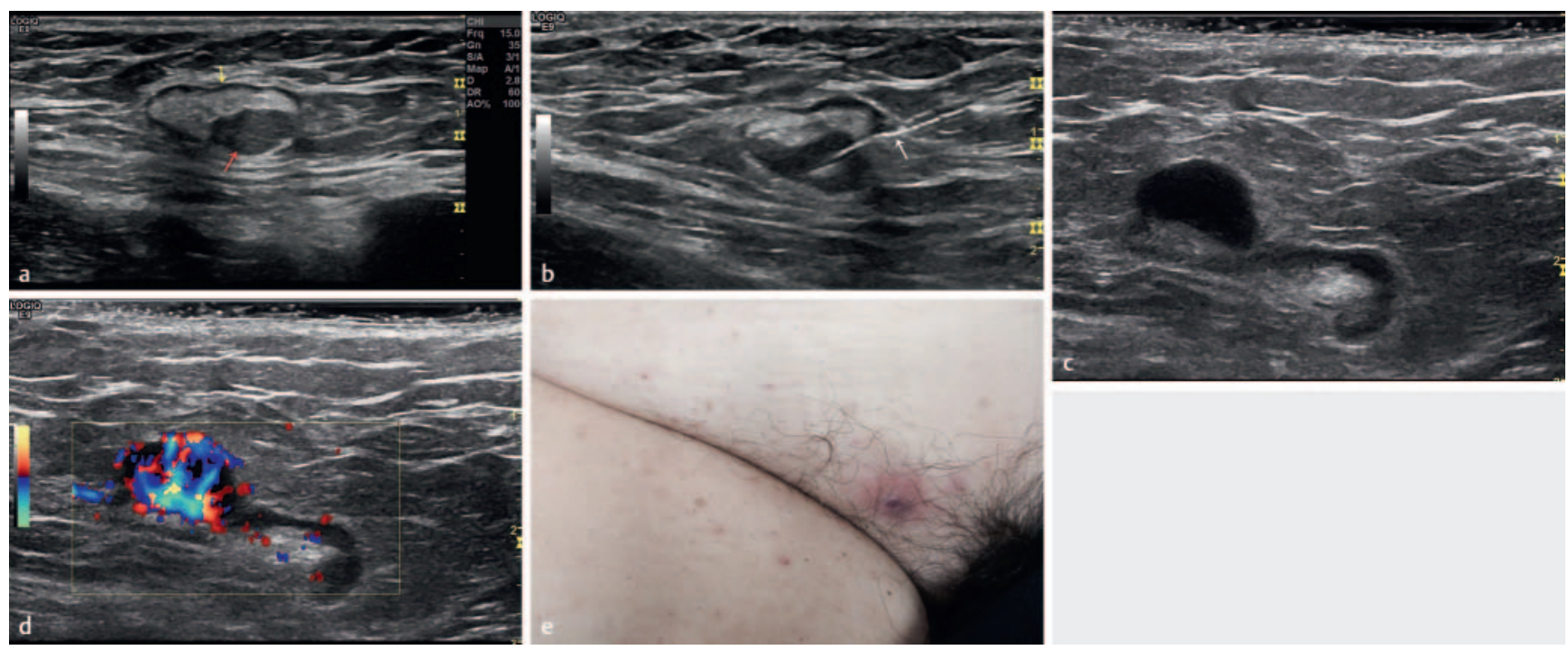

- Abb. 6 a Regionales Verfettungsareal (roter Pfeil), schmaler Kortex (gelber Pfeil). b Nadelreflex (weißer Pfeil) zur zytologischen Sicherung. c, d Regional echoarm verdickter Rindenabschnitt, der in der FKDS hypervaskularisiert mit geordnetem Gefäßbild. e Ursächliche Haarbalgentzündung.

können Tumoren zunächst auf das LK-Zentrum beschränkt sein und die Rindenregion aussparen. Im Unterschied zu entzündlichen und gelegentlich bei lymphomatös transformierten LK sind sie damit oft kugelig, das Verhältnis von Länge zu Tiefendurchmesser ist damit in der Regel zwischen 1 und unter $2[9,10]$. Ein weiteres wichtiges Kriterium betrifft die Tumorvaskularisation und -perfusion. Tumorgefäße haben keine L. muscularis und weisen unterschiedlich große Poren in ihrer Gefäßwand auf. Daher kann Flüssigkeit aus dem Gefäßlumen in das Tumorgewebe des LK abgepresst werden. Dies führt zu einem erhöhten interstitiellen Druck und so zu einer Abnahme der Gewebeperfusion. Das Tumorzentrum wird ischämisch, und letztlich können Nekrosen entstehen $[17,18]$. Wird die Kapsel durch die Tumorinvasion zerstört, kann aus dem Lymphknotengewebe Flüssigkeit ablaufen und zu einem perinodalen Ödem führen ( $\bullet$ Abb.9). 

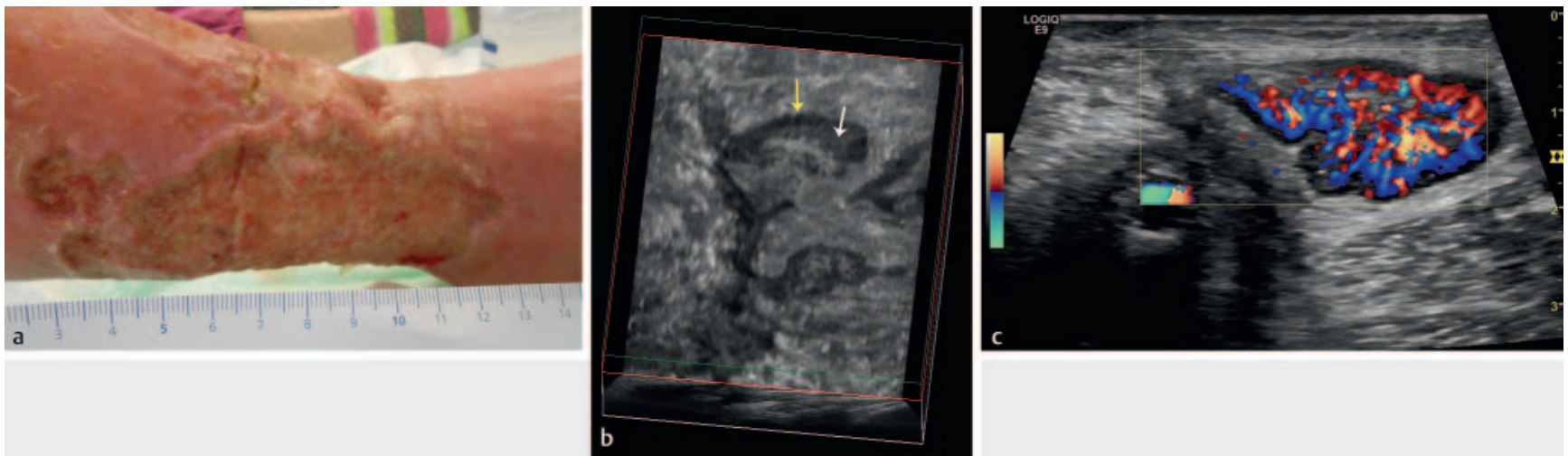

- Abb.7 a Chronisches, fast zirkuläres Unterschenkelulcus. b C-Ebene* mit Darstellung eines von mehreren LK mit Follikeln (gelber Pfeil), weißer Pfeil: Parakortex. c In der FKDS deutliche Hypervaskularisation mit regelrechtem Gefäßbild (baumartig angeordnete Arterien und Venen) C-Ebene: B-Mode-Rekonstruktionsebene parallel zur Kutis aus einem Sweep.
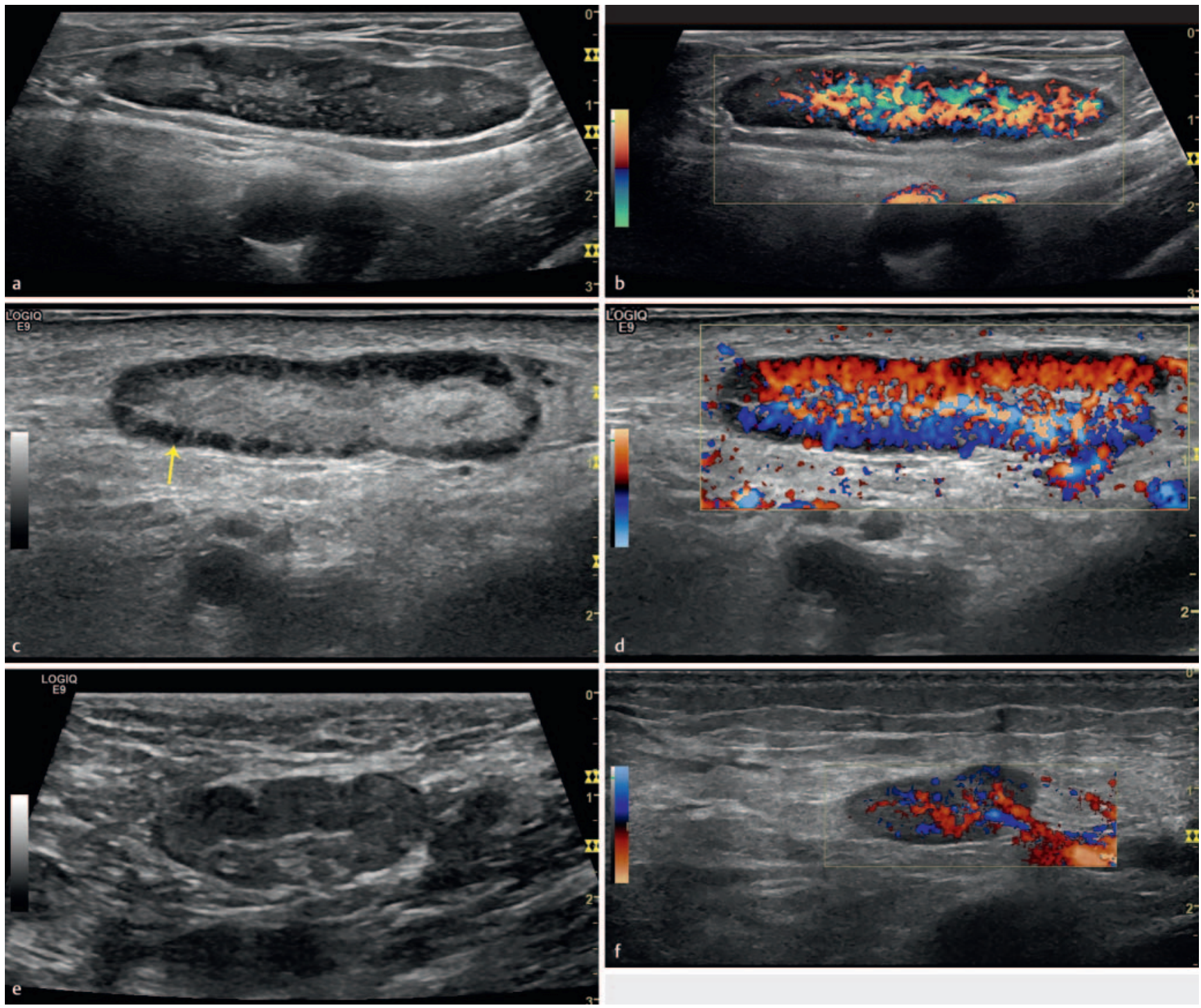

- Abb. 8 Melanompatientin unter Interferontherapie. a Länglicher inguinaler LK mit verdickter Rinde, Solbiati-Index $>2$, und $\mathbf{b}$ vermehrter arterieller und venöser Vaskularisation mit regelrechter Gefäßarchitektur. Histologisch gesicherter reaktiver LK. c Inguinaler LK mit hypertrophierten Follikeln (Pfeil), Solbiati-Index > 2. d Hypervaskularisierter LK mit geordneter arterieller Gefäßarchitektur. e Psoriasispatient mit verdickter Rinde, Solbiati-Index ca. 2, und f vom Hilus aus geordnetem Gefäßbild. 
- Tab.2 Allgemeine Beurteilungskriterien von LK, Charakteristika bei entzündlich reaktiven, lymphomatös und metastatisch transformierten Lymphknoten.

\section{Sonografische Tumorcharakteristika}

\begin{tabular}{|c|c|c|c|}
\hline Modus & \multicolumn{3}{|l|}{ Entzündlich reaktive LK } \\
\hline B-Mode & $\begin{array}{l}\text { Akute E: Meist gleichmäßig verdickte } \\
\text { Rinde, Medulla echoarm. Meist länglich } \\
(\mathrm{LD} / \mathrm{TD}>2) \text {. Zahl \& Größe abnehmend } \\
\text { mit zunehmender Entfernung }\end{array}$ & $\begin{array}{l}\text { Chron. E.: Gleichmäßig } \\
\text { schmale Rinde, echogenes } \\
\text { Zentrum }\end{array}$ & $\begin{array}{l}\text { Akut: Selten perinodal streifige } \\
\text { Flüssigkeit (Ödem) }\end{array}$ \\
\hline FKDS & $\begin{array}{l}\text { Regionale/globale Hyper-vaskularisation, } \\
\text { abnehmend bei chron. E. }\end{array}$ & Arterien- und Venennachweis & Baumartige Gefäßarchitektur \\
\hline \multirow[t]{2}{*}{ CEUS } & $\begin{array}{l}\text { Deutliche, zentrifugal homogene } \\
\text { Hyperperfusion (akute E.) }\end{array}$ & Geringe Perfusion bei chron. E. & $\begin{array}{l}\text { Meist kein perinodaler } \\
\text { Perfusionsnachweis }\end{array}$ \\
\hline & \multicolumn{3}{|l|}{ Lymphom } \\
\hline B-Mode & $\begin{array}{l}\text { Wie entzündlich, ggf. kleinknotige } \\
\text { Musterung im verdickten Rindenanteil } \\
\text { (Follikel), kein echogener Hilus }\end{array}$ & $\begin{array}{l}\text { Multiple, meist bilateral, wei- } \\
\text { tere periphere LK-Stationen } \\
\text { betroffen }\end{array}$ & $\begin{array}{l}\text { Meist kein perinodales Ödem, } \\
\text { abdominelle Mitbeteiligung } \\
\text { (LK und/oder Organe) }\end{array}$ \\
\hline FKDS & Hypervaskularisation & $\begin{array}{l}\text { Meist Arterien- und Venen- } \\
\text { nachweis }\end{array}$ & Baumartige Gefäßarchitektur \\
\hline \multirow[t]{2}{*}{ CEUS } & $\begin{array}{l}\text { Deutliche, zentrifugal homogene } \\
\text { Hyperperfusion }\end{array}$ & $\begin{array}{l}\text { Allenfalls periphere Hypoper- } \\
\text { fusion }\end{array}$ & Meist kein perinodales Ödem \\
\hline & \multicolumn{3}{|l|}{ Karzinom-, Sarkommetastasen } \\
\hline B-Mode & $\begin{array}{l}\text { Knotige Verdickung oder kugelige Form } \\
\text { (LD/TD <2) (Metastasierungsweg?) }\end{array}$ & $\begin{array}{l}\text { Rundlicher echoarm-zysti- } \\
\text { scher LK, kein echogener Hilus }\end{array}$ & Perinodales Ödem bei Kapselinfiltration \\
\hline FKDS & $\begin{array}{l}\text { Geringe bis starke Vaskularisation, ggf. } \\
\text { zentral fehlende Gefäße, Versorgung über } \\
\text { Kapselarterien }\end{array}$ & $\begin{array}{l}\text { Meist chaotische Gefäßarchi- } \\
\text { tektur }\end{array}$ & Meist keine perinodale Vaskularisation \\
\hline CEUS & Geringe bis starke zentripetale Perfusion & $\begin{array}{l}\text { Inhomogene Perfusion, zen- } \\
\text { tral ischämisch-nekrotisch }\end{array}$ & $\begin{array}{l}\text { Perinodale Kontrastierung } \\
\text { (bei kapselüberschreitender Infiltration) }\end{array}$ \\
\hline
\end{tabular}

E: Entzündung, LD: Längsdurchmesser, TD: Tiefendurchmesser, CEUS: Contrast-Enhanced Ultrasound = Ultraschall mit Kontrastmittelverstärkung.

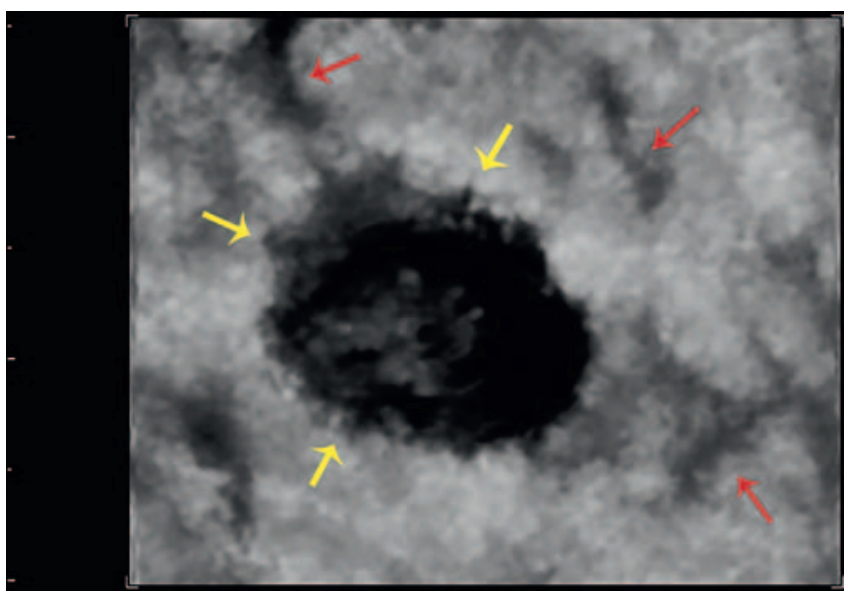

- Abb. 9 Feine, spikulaartige, echoarme Ausläufer über die LK-Kapsel hinaus (gelbe Pfeile) weisen auf eine Tumorinfiltration hin, meist mit begleitendem regionalem Ödem (rote Pfeile).
Der nutritive und $\mathrm{O}_{2}$-Bedarf wird nicht nur über die hilären arteriellen Äste sichergestellt, sondern kann - vor allem bei zentraler Ischämie oder Nekrose - auch über in den LK einstrahlende Kapselarterien erfolgen. ( Abb. 10a-f, 11a, b, 12a, b).

\section{Lymphome}

Bei zufällig entdeckten tumorsuspekten inguinalen Lymphknoten muss entschieden werden, ob es sich um die Manifestation einer Systemerkrankung oder eine regionale LK-Metastase handelt. Dazu sind klinische Daten entscheidend. Bei Kindern und Jugendlichen steht meist eine entzündlich-reaktive Genese im Vordergrund. Feinnadelaspirationsbiopsien (FNAB) werden von einigen Autoren zur Diagnosesicherung empfohlen [19].

Ein isoliert inguinal auftretendes Hodgkin-Lymphom (M. Hodgkin, Stadium I) ist eher selten und fällt durch eine echoarme bis zystische Rindenauftreibung im B-Mode auf. Bei NonHodgkin-Lymphom (NHL) finden sich suspekte LK meist auch an mehreren LK-Stationen, auch eine abdominelle Manifestation (Lymphknoten, Milz-, Leberbeteiligung) ist nicht selten [20]. Eine histologische LK-Begutachtung zur Festlegung des Subtyps wird 

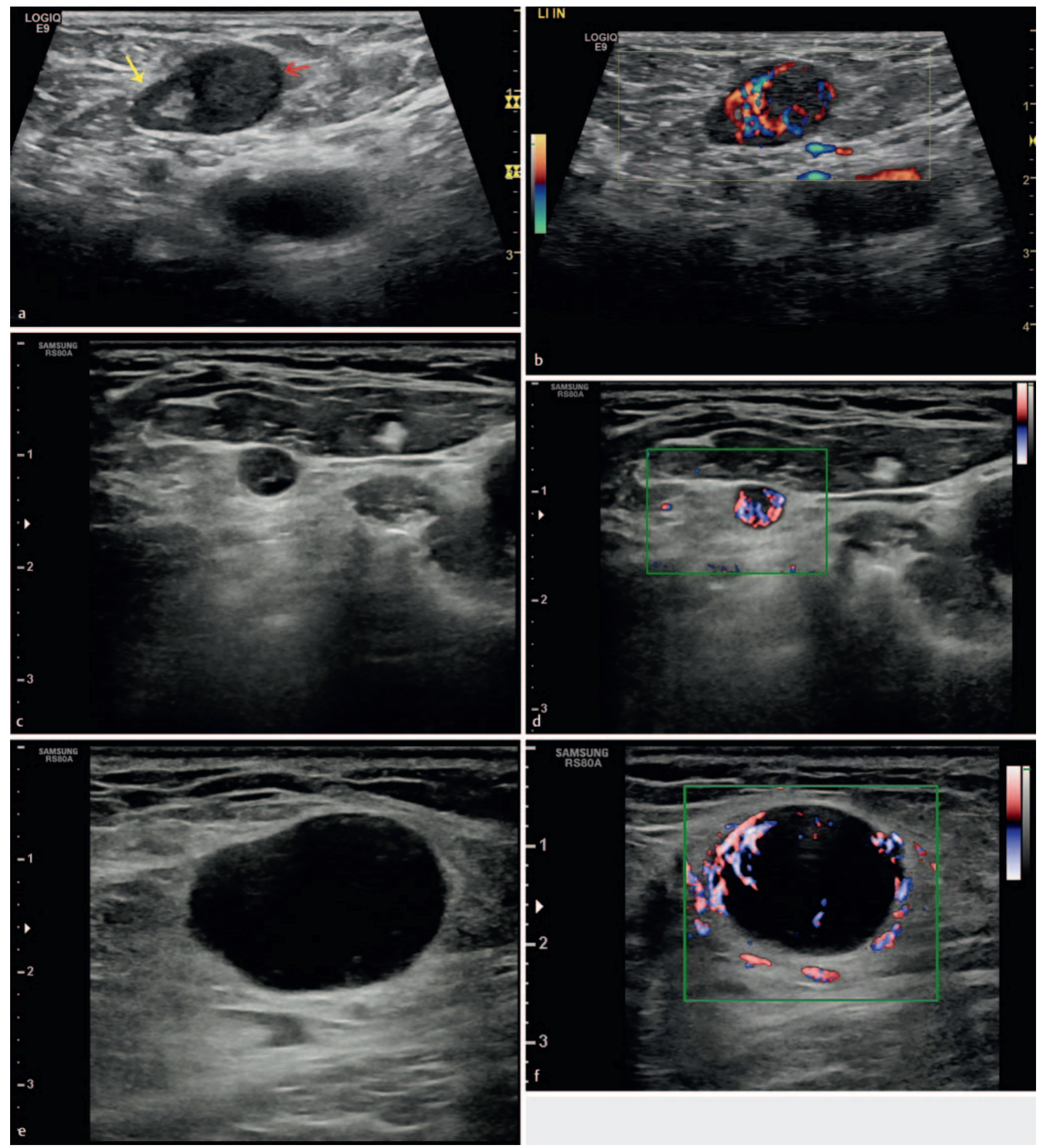

- Abb. 10 a Inguinale Sarkommetastase (gelber Pfeil: normale LK-Rinde, roter Pfeil: etwa $1 \mathrm{~cm}$ große, runde Metastase). b Von peripher einstrahlende Tumorgefäße, ischämisches Tumorzentrum. c $5 \mathrm{~mm}$ große kugelige, echoarm inhomogene und vollständig tumorinfiltrierte Melanommetastase mit d von peripher einstrahlenden Gefäßen, e kugelige, vollständig tumorinfiltrierte, fast zystisch imponierende Melanommetastase mit f zentraler Ischämie und über peripher versorgenden Kapselarterien, zentrale Ischämie. 

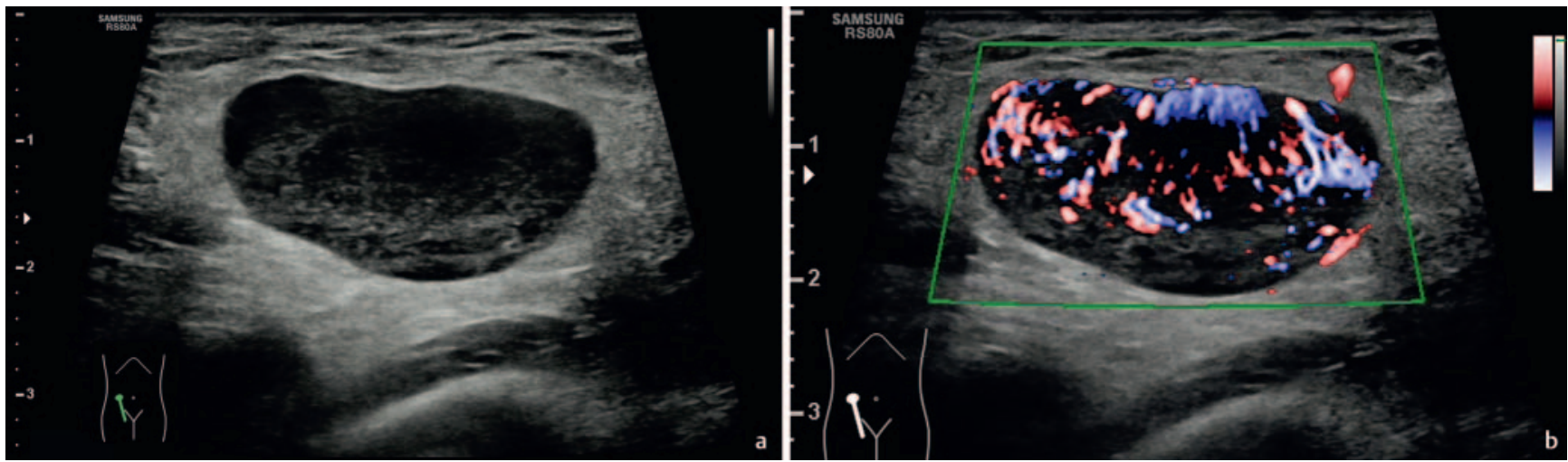

- Abb.11 Inguinale, hypervaskularisierte Melanommetatsase. Solbiati-Index <2. a B-Mode der echoarmen Rinde und der echoarmen, zentral tumorinfiltrierten Medulla. b In der FKDS zeigt sich eine zusätzliche Tumorversorgung über Kaspelarterien.
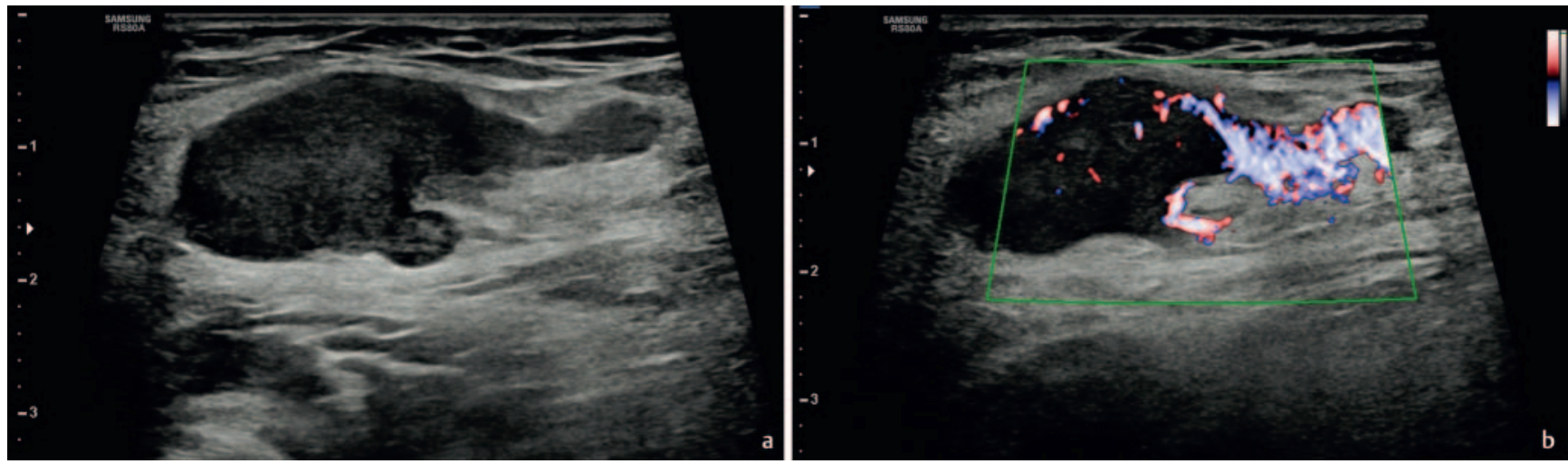

- Abb.12 Zustand nach minimalinvasiv durchgeführter OP eines Zervixkarzinoms. 4 Monate später fand sich eine inguinale LK-Metastase. a Auftreibung des proximalen LK-Endes (links im Bild), das in b eine nur minimale periphere Vaskularisation zeigt, während die wohl noch intakte kaudale Rinde deutlich hypervaskularisiert ist.
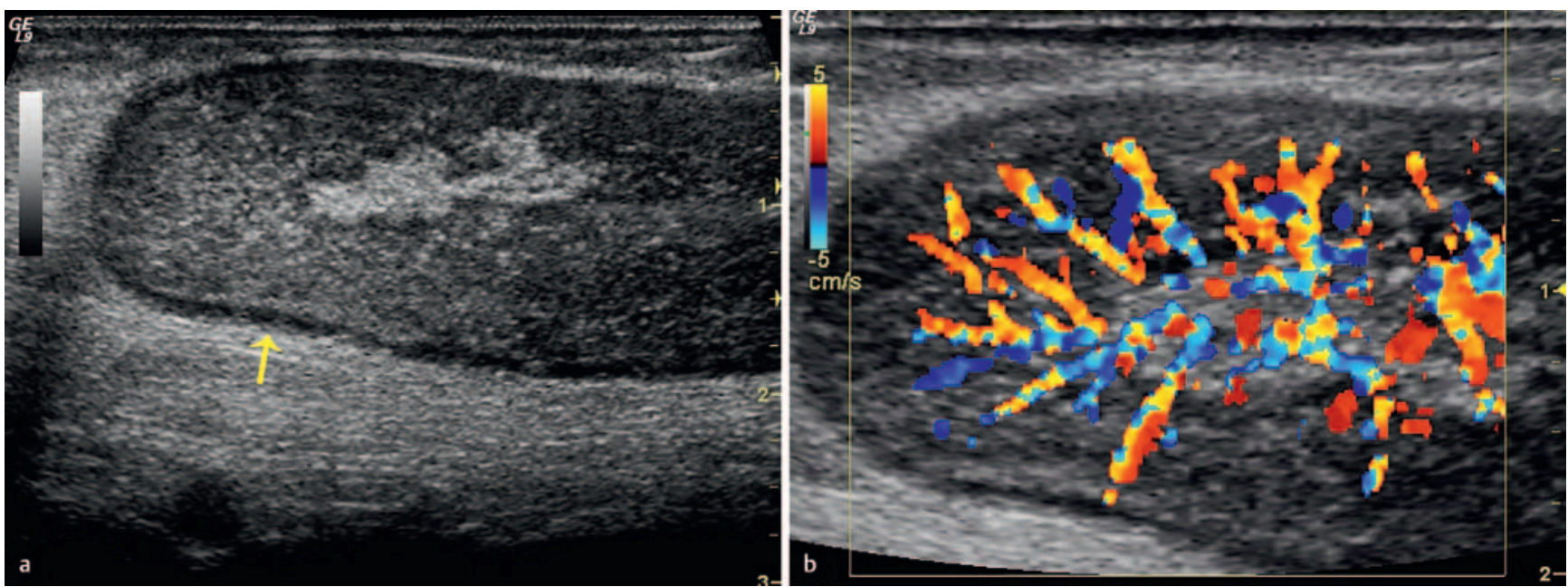

- Abb. 13 B-NHL a. Im B-Mode stellen sich neben einer feinen echoarmen Rinde (Pfeil) multiple feine Knötchen in der Medulla dar. Die FKDS b zeigt eine vermehrte Vaskularisation mit erhaltener Architektur. 

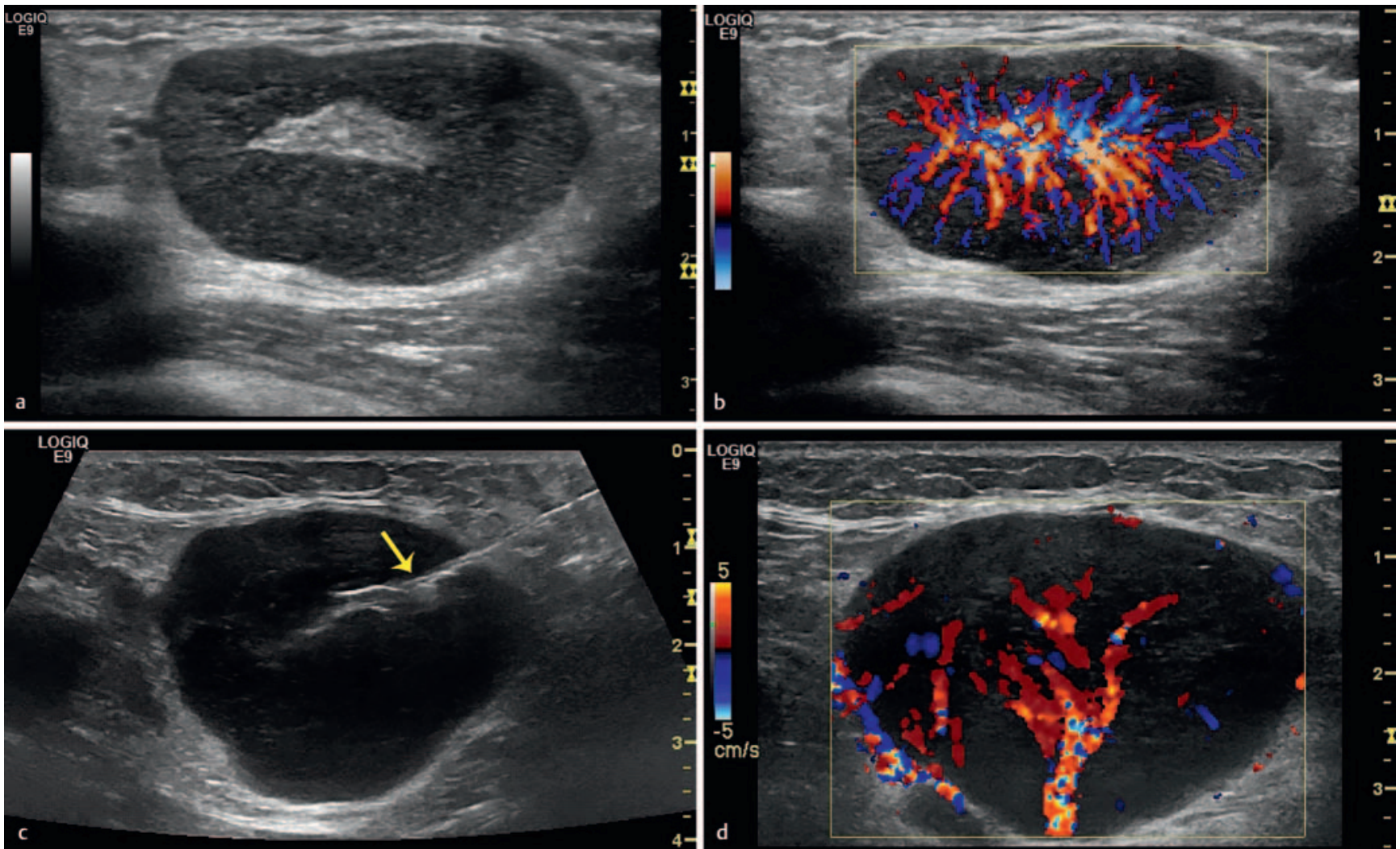

- Abb. 14 Typische periphere LK bei NHL (B-NHL), alle mit einem Solbiati-Index <2. a Im B-Mode echoarme Verdickung der Rinde mit kleinen echoarmen Knötchen und noch vorhandenem echogenem Zentrum. b Hypervaskularisierter LK mit geordneter Gefäßarchitektur. c Rundlicher, echoarmer B-NHL-LK mit Echoreflex der Schneidbiopsienadel (Pfeil), im B-Mode kein Hilus mehr erkennbar. $\mathbf{d}$ Zentrale Gefäßversorgung mit regulärer baumartiger Aufzweigung und geringer peripherer Vaskularisation.
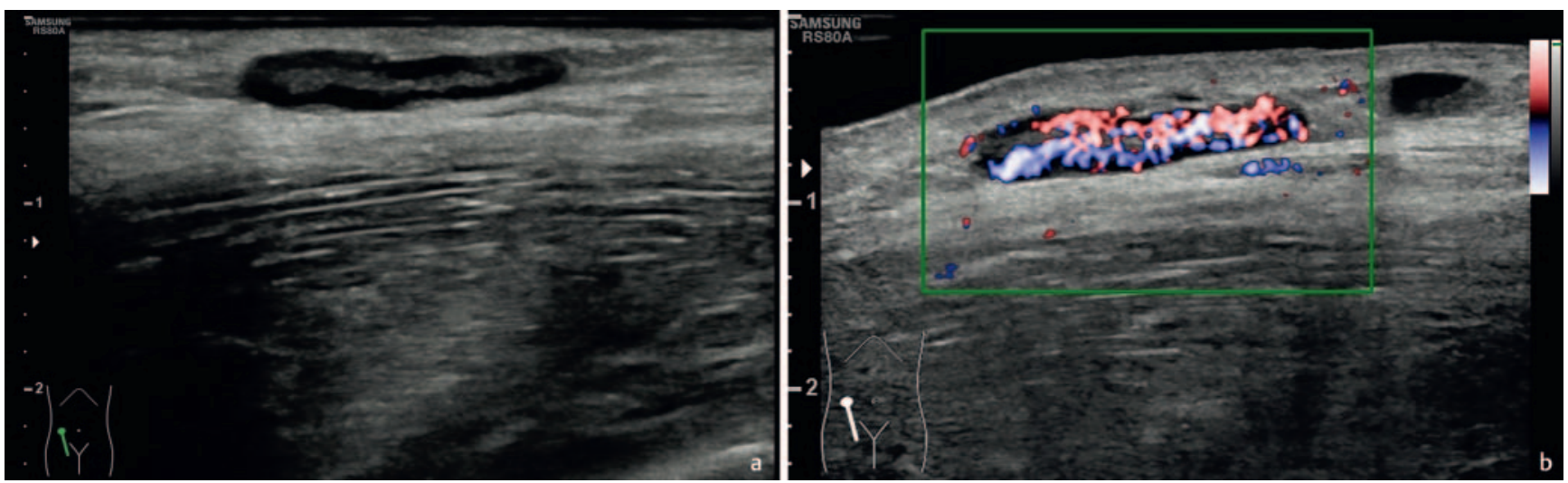

- Abb. 15 56-jähriger Patient mit Mykosis fungoides (T-Zell-Lymphom). a B-Mode eines oberflächlichen länglichen LK (Solbiati-Index > 2) mit erhaltener Architektur (Rinde, Mark, Hilus). b In der FKDS deutlich hypervaskularisierter LK bei geordneter Gefäßarchitektur.

aus onkologischer Sicht meist gefordert, da davon Therapie und Prognose abhängen.

Eine sichere Unterscheidung zwischen Lymphomerkrankung und entzündlich reaktiven LK kann ohne Kenntnis des klinischen Hintergrundes und nur anhand des Ultraschallbefundes eines LK schwierig oder gar unmöglich sein ( $\mathbf{A b b}$. 15). Meist stellen sich Non-Hodgkin-Lymphome als echoarm dar; sie sind stark vaskula- risiert mit baumartiger Gefäßarchitektur. Typischerweise ist ihre Rinde echoarm verdickt, gelegentlich lassen sich kleine hinweisende echoarme Knötchen in der Rinde und der Medulla nachweisen ( Abb. 13a, 14a, 15a). Auch eine komplett zystische Transformation kann ein NHL-typisches sonografisches Merkmal sein. Je nach Subtyp können mehrere betroffene LK bei einem Patienten eine große sonografische Varianz aufweisen. 

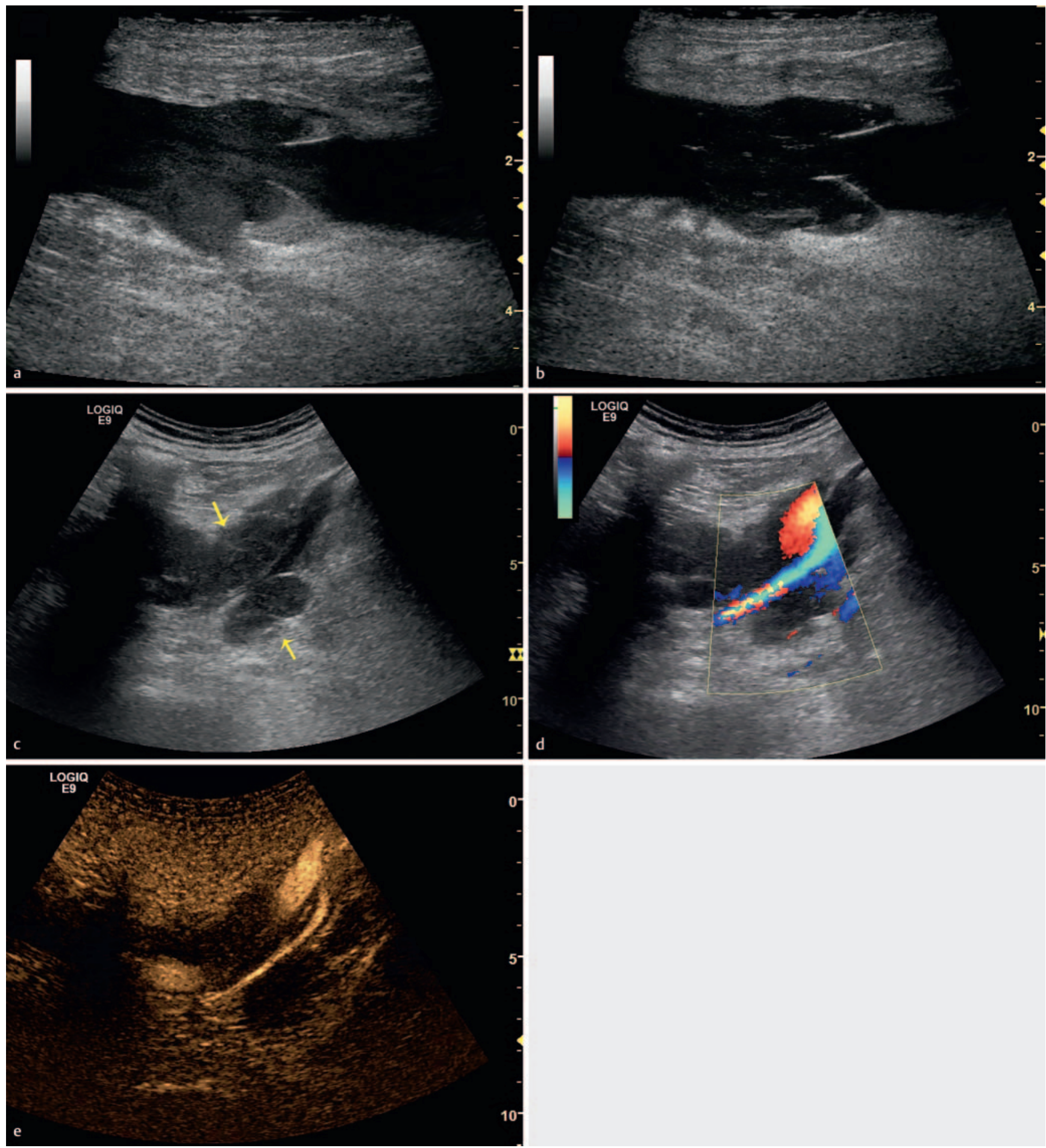

$\mathbf{0}^{-}$

- Abb. 16 a Beckenvenendarstellung bei einseitiger Beinvenendilatation mit Spontanechos an den Venenklappen der proximalen V. femoralis superficialis. b Auflösung der Spontanechos nach Venendekompression c Venöse Kompressionsstenose der V. iliaca externa durch pathologische LK. d Flussbeschleunigung im Stenosebereich (FKDS). e Morphologische Stenosedarstellung im B-Flow-Verfahren.

Da insbesondere Non-Hodgkin Lymphome Gefäße komprimieren können, muss bei einer einseitigen Venendilatation der Beinvenen mit Stasezeichen auch an eine Lymphomerkrankung gedacht werden ( $\vee$ Abb. 16a-c).

\section{Interessenkonflikt}

Die Autorinnen/Autoren geben an, dass kein Interessenkonflikt besteht. 


\section{Literatur}

[1] Willard-Mack CL. Normal Structure, Function, and Histology of Lymph Nodes. Toxicologic Pathology 2006; 34: 409-424

[2] Kubik S, Wirth W. Histology, Anatomy and Lymphograpohic Appearace; Aus: Atlas of Lymphography, Viamonte M, Jr, Rüttimann A. ed. Thieme; 1980

[3] Weskott HP. B-flow-a new method for detecting blood flow. Ultraschall in Med 2000; 21 (2): 59-65. German

[4] Sim JK, Lee JY, Hong HS. Differentiation Between Malignant and Benign Lymph Nodes: Role of Superb Microvascular Imaging in the Evaluation of Cervical Lymph Nodes. J Ultrasound Med 2019; 38 (11): 3025-3036

[5] Weskott HP, loanitescu ES. Diagnostic approach to lymph node diseases in ultrasound; In Dietrich C, ed. In: EUS Course Book; 2012

[6] Weskott HP. Kontrastverstärkte Sonografie in der Lymphknotendiagnostik. Der Radiologe 2018; 58 (6): 563-571

[7] Eigentler TK, Mühlenbein C, Follmann M et al. S3-Leitlinie Diagnostik, Therapie und Nachsorge des Melanoms - Update 2015/2016, Kurzversion 2.0. J Dtsch Dermatol Ges 2017; 15 (6): e1-e41

[8] Dippel E, Assaf C, Becker JC et al. S2k-Leitlinie - Kutane Lymphome Update 2016 - Teil 1: Klassifikation und Diagnostik (ICD10 C82 - C86). J Dtsch Dermatol Ges 2017; 15 (12): 1266-1273

[9] Solbiati L, Rizatto G, Bellotti E et al. High resolution sonography of cervical lymph nodes in head and neck cancer: criteria for differentiation of reactive versus malignant nodes. Radiology 1988; 169: 113

[10] Vassallo P, Wernecke K, Roos $\mathrm{N}$ et al. Differentiation of benign from malignant superficial lymphadenopathy: the role of high-resolution US. Radiology 1992; 183 (1): 215-220
[11] Nagy JA, Chang SH, Dvorak AM et al. Why are tumour blood vessels abnormal and why is it important to know? Br J Cancer 2009; 100 (6): 865-869

[12] Bertolli E, Bevilacqua JL, Molina AS et al. Popliteal sentinel lymph node involvement in melanoma patients. J Surg Oncol 2015; 112 (2): 179-182

[13] Catalano O, Caracò C, Mozzillo N et al. Locoregional spread of cutaneous melanoma: sonography findings. Am J Roentgenol 2010; 194 (3): 735745

[14] Al Hmada Y, Schaefer IM, Fletcher CDM. Hibernoma Mimicking Atypical Lipomatous Tumor: 64 Cases of a Morphologically Distinct Subset. Am J Surg Pathol 2018; 42 (7): 951-957

[15] Bazemore AW, Smucker DR. Lymphadenopathy and malignancy. Am Fam Physician 2002; 66 (11): 2103-2110

[16] Klebl FH, Gelbmann CM, Lammert I et al. Palpatorische und sonografische Detektion von Lymphknotenmetastasen bei lokal fortgeschrittenem malignen Melanom [Detection of lymph node metastases of malignant melanoma by palpation and ultrasound]. Med Klin (Munich) 2003; 98 (12): 783-787

[17] Hobbs SK, Monsky WL, Yuan F et al. Regulation of transport pathways in tumor vessels: role of tumor type and microenvironment. Proc Natl Acad Sci U S A 1998; 95 (8): 4607-4612

[18] Lunt S], Kalliomaki TM, Brown A et al. Interstitial fluid pressure, vascularity and metastasis in ectopic, orthotopic and spontaneous tumours. BMC Cancer 2008; 8: 2

[19] van de Schoot L, Aronson DC, Behrendt $\mathrm{H}$ et al. The role of fine-needle aspiration cytology in children with persistent or suspicious lymphadenopathy. J Pediatr Surg 2001; 36 (1): 7-11

[20] Weskott HP. Ultraschall im klinischen Management maligner Lymphome. Radiologe 2012; 52 (4): 347-359 